\title{
A New Approach to the Detection of Mammogram Boundary
}

\author{
Mohammed Rmili ${ }^{1}$, Abdelmajid El Moutaouakkil ${ }^{2}$, Mousatapha M. Saleck ${ }^{3}$, Maksi Bouchaib ${ }^{4}$, \\ Fatiha Adnani $^{5}$, El Mehdi El Aroussi ${ }^{6}$ \\ ${ }^{1,2,3}$ LAboratory of Research on Optimization of Emergent Systems, Network and Imaging of Computer Science \\ Department of Chouaïb Doukkali University, EL Jadida, Morocco \\ ${ }^{1,5,6}$ LAboratory of Mathematics Applied on Physics and Industry, Mathematics Department of Chouaïb Doukkali University, \\ EL Jadida, Morocco \\ ${ }^{4}$ Department of Radiology, Hospital Mohammed V, Eljadida, Morocco
}

\begin{tabular}{l} 
Article Info \\
\hline Article history: \\
Received Jan 5, 2018 \\
Revised Apr 15, 2018 \\
Accepted Apr 22, 2018 \\
\hline
\end{tabular}

Keyword:

Line segment

Mammography

Mass segmentation

Region growing algorithm

\begin{abstract}
Mammography is a method used for the detection of breast cancer. computer-aided diagnostic (CAD) systems help the radiologist in the detection and interpretation of mass in breast mammography. One of the important information of a mass is its contour and its form because it provides valuable information about the abnormality of a mass. The accuracy in the recognition of the shape of a mass is related to the accuracy of the detected mass contours. In this work we propose a new approach for detecting the boundaries of lesion in mammography images based on region growing algorithm without using the threshold, the proposed method requires an initial rectangle surrounding the lesion selected manually by the radiologist (Region Of Interest), where the region growing algorithm applies on lines segments that attach each pixel of this rectangle with the seed point, such as the ends (seeds) of each line segment grow in a direction towards one another. The proposed approach is evaluated on a set of data with 20 masses of the MIAS base whose contours are annotated manually by expert radiologists. The performance of the method is evaluated in terms of specificity, sensitivity, accuracy and overlap. All the findings and details of approach are presented in detail.
\end{abstract}

Copyright () 2018 Institute of Advanced Engineering and Science. All rights reserved.

\section{Corresponding Author: \\ Mohammed Rmili, \\ Department of Computer Science, University Chouaïb Doukkali, EL Jadida, Morocco. \\ Email: gmrmili@gmail.com}

\section{INTRODUCTION}

Currently breast cancer is the second cause of women's death . Cancer affects women between forty and fifty-five years old [1]. It attacks women most [2]. Unfortunately, there is still no prevention and the solution lies in the early detection to increase the effectiveness of treatment and reduce the risk of mortality.

In breast cancer setting, the effectiveness of this pathology treatment have need the early detection. At present Mammography is the most effective technique for the early diagnosis of breast cancer [3]. Diagnostic support of techniques is being developed to facilitate the work of radiologists. According to this vision, for the last years Computer-Assisted Diagnosis became very important in the international research in the world [4].

Detection and segmentation of the mass in mammography from background tissue is a major problem. especially, finding a correct mass edge is the major key for good mass interpretation, because mass interpretation depends on the edge, margin and shape characteristics of a mammary mass. 
In mammographic diagnosis, non-cancerous lesions can be misinterpreted as cancer (false positive value), while cancers can be misinterpreted as non-cancerous lesions (false negative value). Correspondingly, radiologists fail to detect $10 \%$ to $30 \%$ of breast cancers [5]-[7]. Computer-aided detection (CAD) systems have been developed to reduce the cost and improve the ability of medical images interpretation and distinguish between benign and malignant tissues[8]-[10].

Such a CADx system requires that the mass limit be detected as accurately as possible. Consequently he segmentation algorithm should detect an accurate limit of the mass. in CADx systems the accuracy of the segmentation of the breast mass contour plays an important role in mass classification, because since most of the crucial properties of the mammary masses that define malignancy are related to its morphology. Consequently, the malignant masses form irregular forms; Conversely, the benign masses form regular forms.

Alsarori et al [11], have applied the Multiple-Thresholding method OTSU to segment the region of interest (ROI). Then The texture characteristics of the segmented ROI which are used to classify the ROI as abnormal or normal tissue by using a Neural Network, Information (ANN). Anand et al [12], used a hybrid of Fuzzy c- Means algorithm and Self Organizing Map algorithm to segment the breast image and then categorize the tumour: affected breast images and normal breast images. Shareef [13] applied an algorithm based on the morphological operation and segmentation watershed transformation. This approach has obtained a very similar diagnosis of breast tumour in types of medical images [14], proposed a new algorithm segmentation to improve the contour of a mass of a given region of interest based on the region growing algorithm with the ability to adaptively adjust the threshold value. Vedanarayanan et al [15], propose a segmentation technique based on modified expectations Maximization and Modified Snake Algorithm to isolate the abnormality. And for describing abnormality, this approach uses the following features: Area, Minor Axis Length, Major Axis Length, Perimeter, Orientation, Centroid, Eccentricity, EquivDiameter, Solidity and convex area. The back propagation network is used to determine the presence of cancer. S. M. L. de Lima et al [16], proposed a method for detecting and classifying breast lesions using feature extraction based on the Calculation of Zernike Moments from a series of multi-resolution image components obtained by the series of wavelets. Chaghari et al [17], presented a new method to detect the mass in the mammogram based on cellular learning automata algorithm. mammogam has low contrast of microcalcifications and noise, K. Taifi et al [18], proposed a hybrid method ,to enhance the contrast of a mammography image, combining contourlet and homomorphic filtering. Mustafa et al [19], presents a method for segmenting lesions using the active Chan-Vese contour and the localized active contour. then, the effectiveness of these both methods are compared and chosen to be the best method.

In this work, a new method to segment the contour of masses is designed based on Region Growing Algorithm that is applied in this research on each line segment that attach each pixel of the rectangle to the seed point, which presents the region of interest.

This paper is organized as follows. In Section (2), we present the methodology for mass segmentation. Section (3) involves some experiments to verify and discuss the proposed method. Conclusion and future work is discussed in Section (4).

\section{METHODOLOGY}

In this section, we describe the steps of the proposed methodology: image acquisition, pretreatment, and Mass accurate segmentation. The proposed methodology of breast mass segmentation can be schematically described in Figure 1.

\subsection{Image acquisition}

The credit of the mammograms provided in this work are taken from Mammography Image Analysis Society (MIAS) [20]. The MIAS offered some corresponding information of lesion area such as type, location, severity, central coordinate and radius by experts and each image is $1024 \times 1024$ pixels.

\subsection{Pretreatment}

Mammograms are highly noisy images. Since our approach is based on pixel intensities, noise may distort the results. So, a filtering operation is required.

In our proposed method the first step involves pretreatment using Median Filter, It is a nonlinear filter which is efficient in removing salt and pepper noise tends to keep the sharpness of image edges while removing noise [21]. 


\subsection{Neighborhood}

When processing an image, is necessary to add a definition of the neighborhood between the pixels. In the $2 \mathrm{D}$ case, it is necessary to specify whether a pixel is considered has neighbors the pixels which have one side in common with it alone or also those that share a vertex with it.

In our work, two pixels are considered neighbors if they have at least one corner in common, so that each pixel is not at the edge of the image with 8 neighboring pixels, which is the maximum.

\subsection{Breast mass contour segmentation algorithm}

In this approach we centre on detection the mass limits. We propose a new breast mass segmentation method for a given ROI in mammographic image. This method requires a mass-limiting rectangle that is drawn manually by the user and given to the system as input. in this work We do not prefer to use the result of a method of segmentation, because false positives can proceed in machine segmentation techniques [22]

The detection of breast mass in a mammographic image is based on the law that the pixels within a mass have different characteristics from the other pixels that surrounding the mass. These characteristics may be associated to the intensity of the gray level, the morphological characteristics or the texture.

Methods of breast mass segmentation can be classified into three groups: contour-based, regionbased and group-based. Contour-based methods depend on the boundary of regions, while Region-based methods divide the image into spatially connected homogeneous regions and, the grouping methods arrange the pixels that have the same properties and can product unconnected regions. Given that we are aiming to improve a region's mass limit, we propose an improvement in the region growing approach, which is one of the known methods in the region.

In our approach the initial points are the points of the rectangle and its center. Our segmentation approach widens each region or an initial point by neighboring pixels similar in one direction on a line segment so that the ends of each line segment widen in a direction towards one another, the process stops when each pixel in the line segment binds to a region, without using the threshold. A similar term means a pixel whose Euclidean distance from its intensity to mean of the region is minimal.

In the following, we present the steps of our method such as $S=\left\{S_{1} \ldots S_{n}\right\}$ the pixels of the rectangle, $S_{0}$ its center and $\lambda\left(\mathrm{p}_{\mathrm{i}}\right)$ the Euclidean distance between pi and are closer centroid to the group, Pred $\left(p_{i}\right)$ be the predecessor of a pixel $p_{i}$ and $V(p)$ be the intensity of gray level of the the pixel $p$.

Step 1: Median filter is applied to remove the noise from mammography image.

Step 2: in this step, a rectangle surrounding the mass has been created, and then for each pixel of the rectangle $\mathrm{Si}$ and its center $\mathrm{S}_{0}$ a line segment $\left[\mathrm{S}_{0}, \mathrm{~S}_{\mathrm{i}}\right]$ will be created.

Step 3: In this step we apply the algorithm 1 on each line segment.

Algorithm 1: Algorithm of Segmentation

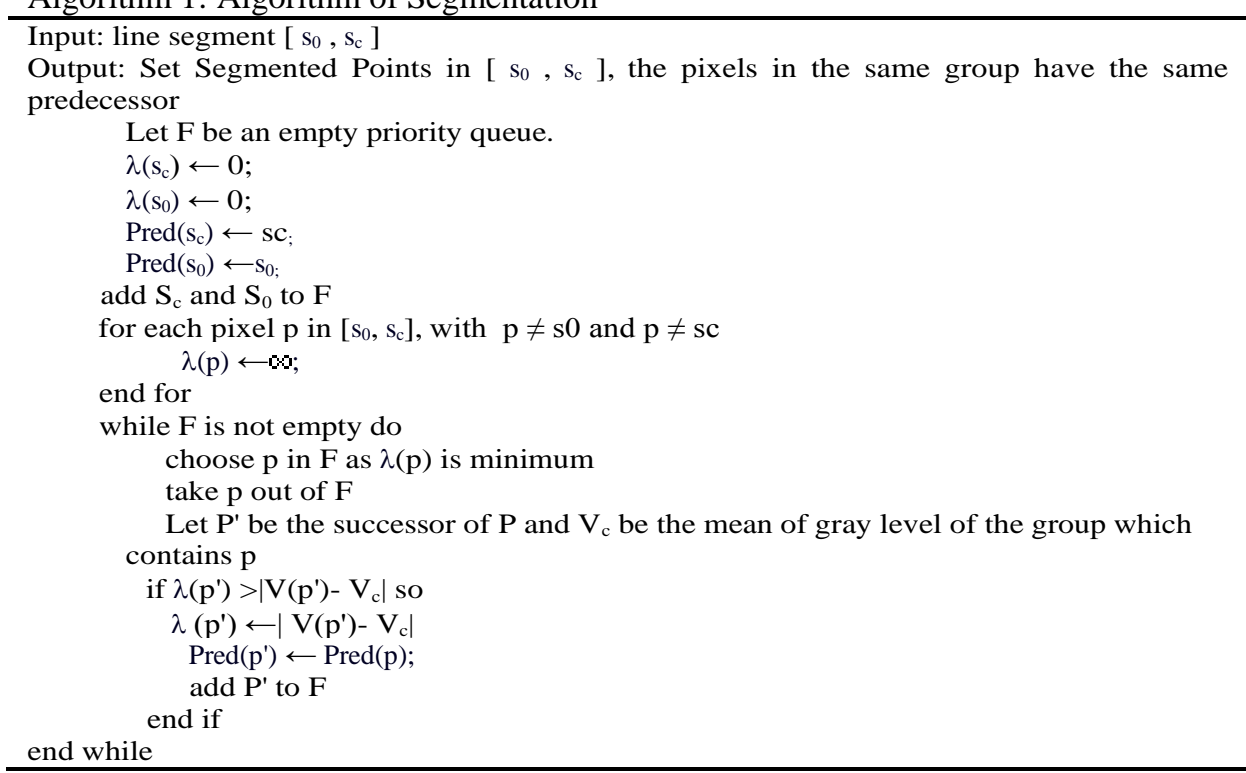




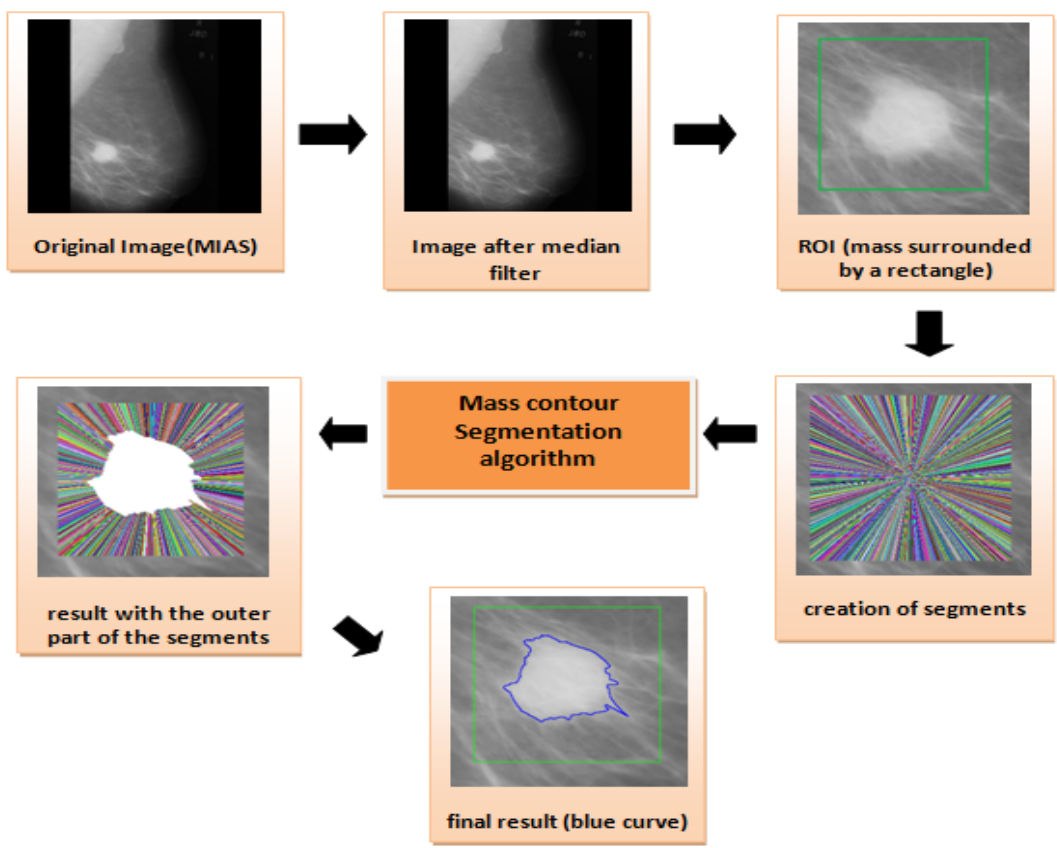

Figure 1. Block diagram of mass segmentation methodology

\section{EXPERIMENT RESULTS AND DISCUSSION}

Our proposed method was tested on 20 mammograms with abnormal mammary regions from the MIAS database. The experimental results are shown in Figure 3.

\subsection{Validation measures}

The results are evaluated using performance measures: specificity, sensitivity, accuracy and overlap, can be calculated using the number of samples correctly classified as follows.

$$
\begin{aligned}
& \text { Sensitivity (SN) }=\frac{\mathrm{TP}}{\mathrm{TP}+\mathrm{FN}} \\
& \text { Specificity (SP) }=1-\frac{\mathrm{FP}}{\mathrm{FP}+\mathrm{TN}} \\
& \text { Accuracy (AC) }=\frac{\mathrm{TP}+\mathrm{TN}}{\mathrm{TP}+\mathrm{FP}+\mathrm{FN}+\mathrm{TN}} \\
& \text { Overlap(AOM) }=\frac{\mathrm{TP}}{\mathrm{TP}+\mathrm{FP}+\mathrm{FN}}
\end{aligned}
$$

where TP number of true positive ,FP number of false negative, FN number of false negative, TN number of true negative(see Figure 2).

\subsection{Segmentation results}

The comparisons of the segmentation results between the proposed method and the manually segmented image by radiologist are shown in Figure 3. In Figure 3, the blue contours are the segmentation results using the proposed algorithm and the black contours are the results obtained by a radiologist. From Figure 3, we can find that the proposed method can obtain good results. Table 1 shows the results of quantitative analysis and from the results we can also prove the effectiveness of the proposed algorithm. The proposed method has a few limitations such as the object to be segmented is already ROI images that have been previously selected in whole mammograms. Thus, a mass detection step must be merged into the algorithm in the future.

The proposed method provides average sensitivity of 0.83 , average specificity of 0.97 , accuracy of 0.91 and overlap of 0.79 . 


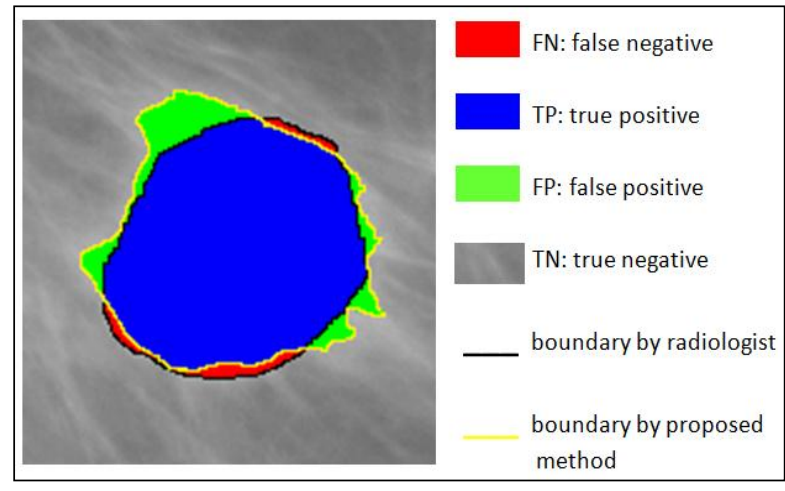

Figure 2. True positive, false positive, true negative and false negative definition

Table 1. Validation Measure Data of 20 Masses

\begin{tabular}{ccccc}
\hline Image & Sensitivity & Specificity & Accuracy & Overlap \\
\hline mdb010 & 0,91 & 0,98 & 0,96 & 0,87 \\
mdb005 & 0,84 & 0,98 & 0,93 & 0,82 \\
mdb012 & 0,81 & 0,98 & 0,91 & 0,78 \\
mdb015 & 0,80 & 1,00 & 0,91 & 0,80 \\
mdb021 & 0,95 & 0,99 & 0,98 & 0,93 \\
mdb023 & 0,82 & 0,98 & 0,94 & 0,80 \\
mdb025 & 0,85 & 0,98 & 0,95 & 0,80 \\
mdb028 & 0,95 & 0,97 & 0,96 & 0,90 \\
mdb069 & 0,78 & 0,94 & 0,88 & 0,71 \\
mdb092 & 0,81 & 0,91 & 0,85 & 0,67 \\
mdb097 & 0,70 & 0,96 & 0,83 & 0,60 \\
mdb132 & 0,98 & 0,98 & 0,98 & 0,89 \\
mdb132 & 0,82 & 1,00 & 0,94 & 0,81 \\
mdb134 & 0,84 & 0,99 & 0,94 & 0,84 \\
mdb142 & 0,89 & 0,97 & 0,96 & 0,77 \\
mdb144 & 0,71 & 0,97 & 0,87 & 0,67 \\
mdb202 & 0,84 & 0,99 & 0,93 & 0,82 \\
mdb267 & 0,75 & 0,99 & 0,89 & 0,74 \\
mdb271 & 0,69 & 0,81 & 0,71 & 0,68 \\
mdb184 & 0,96 & 0,94 & 0,95 & 0,86 \\
Average & 0,83 & 0,97 & 0,91 & 0,79 \\
\hline
\end{tabular}

Results obtained from our approach are compared with other existing methods for breast segmentation, Table 2 shows the results of this comparison.

Table 2. Performance Comparison

\begin{tabular}{|c|c|c|c|c|c|}
\hline \multirow{2}{*}{$\begin{array}{c}\text { Paper } \\
\text { Reference }\end{array}$} & \multirow{2}{*}{ Proposed Method } & \multicolumn{4}{|c|}{ Results } \\
\hline & & Accuracy & Specificity & Sensitivity & Overlap \\
\hline [23] & Marker-Controlled Watershed and Morphological gradient & -- & -- & -- & 0.72 \\
\hline [24] & BHEA- EDA- BBDA- PMDA- ASB- SRGA & 0.99 & 0.92 & 0.94 & -- \\
\hline [25] & Mean shift and Iris filter & -- & -- & 0.81 & 0.60 \\
\hline \multirow[t]{2}{*}{ [26] } & Region Growing & -- & -- & -- & 0.83 \\
\hline & Dynamic Programming-Based & -- & -- & -- & 0.72 \\
\hline Proposed & Region Growing & 0,91 & 0,97 & 0,83 & 0,79 \\
\hline
\end{tabular}




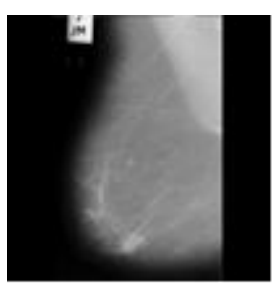

(a)

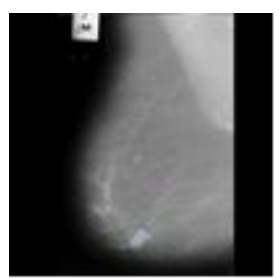

a1

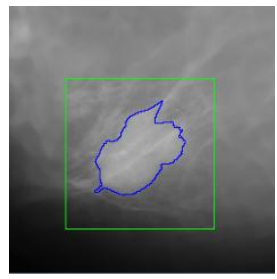

a1-enlarge

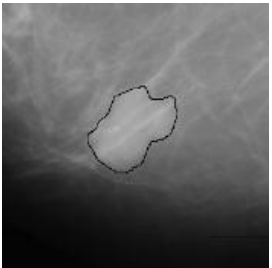

a2

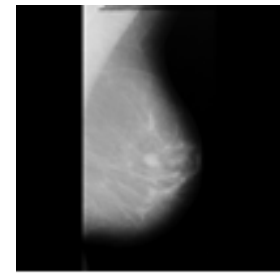

(b)

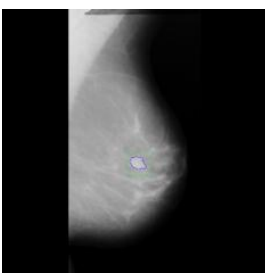

b1

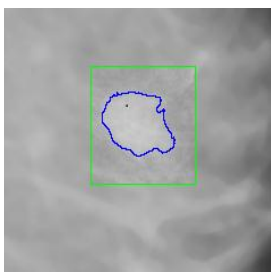

b1-enlarge

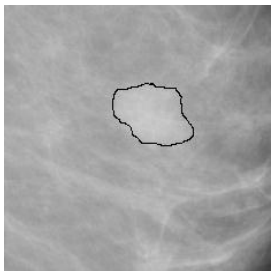

b2

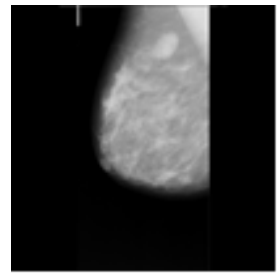

(c)

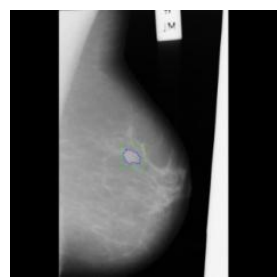

c1

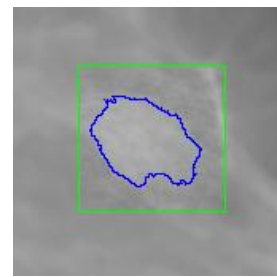

c1-enlarge

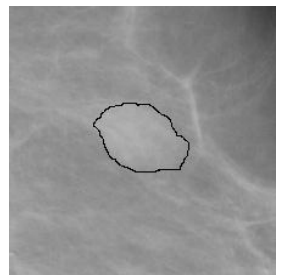

c2

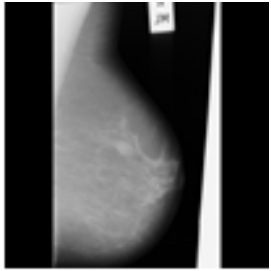

(d)

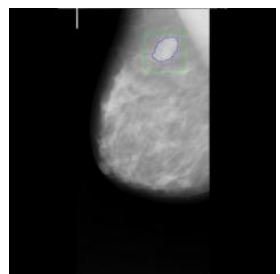

d1

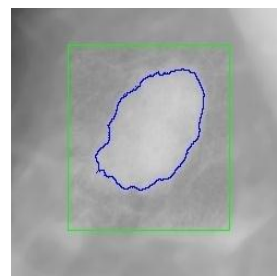

d1-enlarge

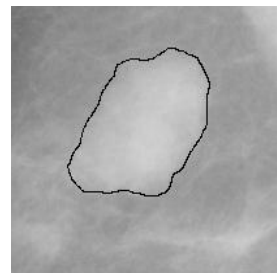

d2

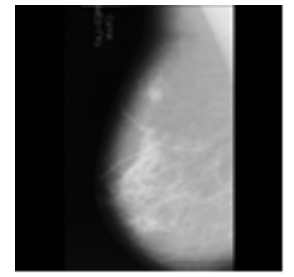

(e)

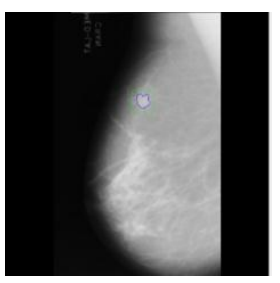

e1

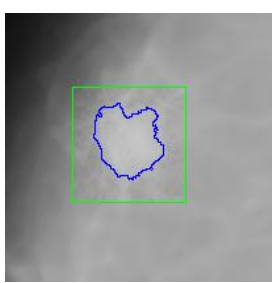

e1-enlarge

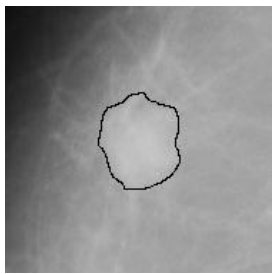

e2

Figure 3. Experimental results of mammograms from MIAS database: (a)-(e) Original images, (a-1)-(e-1) Results by the proposed method, (a-2)-(e-2) The ground truth

\section{CONCLUSION}

In this work, we present a new approach to the detection of mammogram boundary for a given ROI in mammograms. The approach is an extended version of the region growing algorithm .

The proposed approach is evaluated using a reference mammography data set MIAS, where expert radiologists have chosen boundaries. The performance of the method is evaluated using performance measures: specificity, sensitivity, accuracy and overlap .we developed a data set containing 20 masses. We have shown that our method gives good results.

The margins, contour and shape of mass implicate valuable information to determine the severity of the mammary mass. so It is important to find the mass as precise as possible. After segmentation of the breast mass area accurately, the properties of the segmented mass can be extracted and analyzed to determine mass malignancy. It is sure that the quality of the mass properties extracted depends on the success rate of the mass segmentation algorithms used. In the future, we plan to analyze the segmented mass to extract more descriptive and informative information that can be used to interpreted the mammary mass.

\section{REFERENCES}

[1] S. Buseman, J. Mouchawar, N. Calonge, and T. Byers, "Mammography Screening Matters for young women with Breast Carcinoma: Evidence of Downstaging among 42-49-year-old women with a History of Previous 
Mammography Screening”, Cancer, vol. 97, no. 2, pp. 352-358, 2003.

[2] C. Ho, D. Hailey, R. Warburton, J. H. MacGregor, E. D. Pisano, and J. Joyce, "La mammographie numérique comparativement à la mammographie sur film avec écran: évaluation technique, clinique et économique", Rapp. off. Can. Coord. l'évaluation des Technol. la santé, 2002.

[3] G. Xu, K. Li, And G. Feng, "Comparison of Three Imaging Methods in the Early Diagnosis of the Breast Cancer [J]", J. Cap. Med. Univ., vol. 3, p. 11, 2009.

[4] O. Cheng, D. Hui, and W. Guangzhi, "Segmentation of Masses in Mammograms", Beijing Biomed. Eng., 2007.

[5] M. L. Giger, "Computer-aided Diagnosis in Radiology", Elsevier, 2002.

[6] K. Kerlikowske et al., "Performance of Screening Mammography among women with and without a first-degree relative with Breast cancer", Ann. Intern. Med., vol. 133, no. 11, pp. 855-863, 2000.

[7] R. E. Bird, T. W. Wallace, and B. C. Yankaskas, "Analysis of cancers missed at screening Mammography", Radiology, vol. 184, no. 3, pp. 613-617, 1992.

[8] C. J. Vyborny, M. L. Giger, and R. M. Nishikawa, "Computer-Aided Detection and Diagnosis of Breast cancer", Radiol. Clin. North Am., vol. 38, no. 4, pp. 725-740, 2000.

[9] K. Doi, H. MacMahon, S. Katsuragawa, R. M. Nishikawa, and Y. Jiang, "Computer-aided Diagnosis in Radiology: Potential and Pitfalls", Eur. J. Radiol., vol. 31, no. 2, pp. 97-109, 1999.

[10] M. L. Giger, N. Karssemeijer, and S. G. Armato, "Guest Editorial Computer-aided Diagnosis in Medical Imaging", IEEE Trans. Med. Imaging, vol. 20, no. 12, pp. 1205-1208, 2001.

[11] F. A. S. Alsarori and R. Hassanpour, "Automatic Detection of Breast cancer in Mammogram Images", $J . A d v$. Technol. Eng. Res., vol. 2, no. 6, pp. 196-201, 2016.

[12] S. Anand, V. Vinod, and A. Rampure, "Application of Fuzzy c-means and Neural Networks to Categorize Tumor Affected Breast MR Images", Int. J. Appl. Eng. Res., vol. 10, no. 64, p. 2015, 2015.

[13] S. R. Shareef, "Breast cancer Detection based on Watershed Transformation", vol. 11, no. 1, pp. 237-245, 2014.

[14] T. Berber, A. Alpkocak, P. Balci, and O. Dicle, "Breast mass Contour Segmentation Algorithm in Digital Mammograms", Comput. Methods Programs Biomed., vol. 110, no. 2, pp. 150-159, 2013.

[15] V. Vedanarayanan and N. M. Nandhitha, "Advanced Image Segmentation Techniques for Accurate Isolation of Abnormality to Enhance Breast cancer Detection in Digital Mammograph", Biomed. Res., vol. 28, no. 6, pp. 2753-2757, 2017.

[16] S. M. L. de Lima, A. G. da Silva-Filho, and W. P. dos Santos, "Detection and Classification of Masses in Mammographic Images in a Multi-kernel Approach”, Comput. Methods Programs Biomed., vol. 134, pp. 11-29, 2016.

[17] E. Chaghari and A. Karimi, "A Novel Approach for Tumor Detection in Mammography Images," TELKOMNIKA (Telecommunication, Computing, Electronics and Control), vol. 12, no. 8, pp. 6211-6216, 2014.

[18] K. Taifi, R. Ahdid, M. Fakir, and S. Safi, "A Hybrid the Nonsubsampled Contourlet Transform and Homomorphic Filtering for Enhancing Mammograms”, vol. 16, no. 3, pp. 539-545, 2015.

[19] M. Mustafa, H. Najwa Omar Rashid, N. Rul Hasma Abdullah, R. Samad, and D. Pebrianti, "Mammography Image Segmentation: Chan-Vese Active Contour and Localised Active Contour Approach", Indones. J. Electr. Eng. Comput. Sci., vol. 5, no. 3, p. 577, 2017.

[20] J. Suckling et al., "The Mammographic Image Analysis Society Digital Mammogram Database", Expert. Medica, Int. Congr. Ser., vol. 1069, pp. 375-378, 1994.

[21] J. Pragathi, "Segmentation Method for ROI Detection in Mammographic Images u sing Wiener Filter and Kittler' s Method", vol. 2013, pp. 27-33, 2013.

[22] A. Rojas-Domínguez and A. K. Nandi, "Development of Tolerant Features for Characterization of Masses in Mammograms", Comput. Biol. Med., vol. 39, no. 8, pp. 678-688, 2009.

[23] S. Xu, H. Liu, and E. Song, "Marker-Controlled Watershed for Lesion Segmentation in Mammograms", pp. 754-763, 2011.

[24] I. K. Maitra, S. Nag, and S. K. Bandyopadhyay, "Automated Digital Mammogram Segmentation for Destection of Abnormal Masses using Binary Homogeneity Enhancement”, Indian J. Comput. Sci. Eng., vol. 2, no. 3, pp. 416-427, 2011.

[25] T. Terada, Y. Fukumizu, H. Yamauchi, H. Chou, and Y. Kurumi, "Detecting Mass and its Region in Mammograms Using Mean Shift Segmentation and Iris Filter", pp. 1176-1179, 2010.

[26] A. R. Domínguez and A. K. Nandi, "Toward Breast cancer Diagnosis based on Automated Segmentation of Masses in Mammograms", vol. 42, pp. 1138-1148, 2009. 